\title{
A Speedy Recovery with Medical Management in a Patient with Emphysematous Gastritis
}

Tanvi Khurana, MD

\section{INTRODUCTION}

Emphysematous gastritis is a rare and serious condition characterized by evidence of intramural air and inflammation of the gastric wall as well as systemic toxicity. It is generally caused by local infection by gas-forming organisms through a mucosal defect or via hematogenous spread from a distant focus. ${ }^{1}$ Since emphysematous gastritis has a fulminant course with a mortality rate of $60 \%$, prompt recognition as well as early treatment are crucial. ${ }^{2}$ Here, we present a case of a 65 year-old male who presented with abdominal pain and had CT findings consistent with emphysematous gastritis. He was treated with antibiotics and had a swift recovery.

\section{CASE PRESENTATION}

A 65-year-old Caucasian man with past medical history of insulin-dependent diabetes, hypertension, hyperlipidemia, coronary artery disease (with history of three

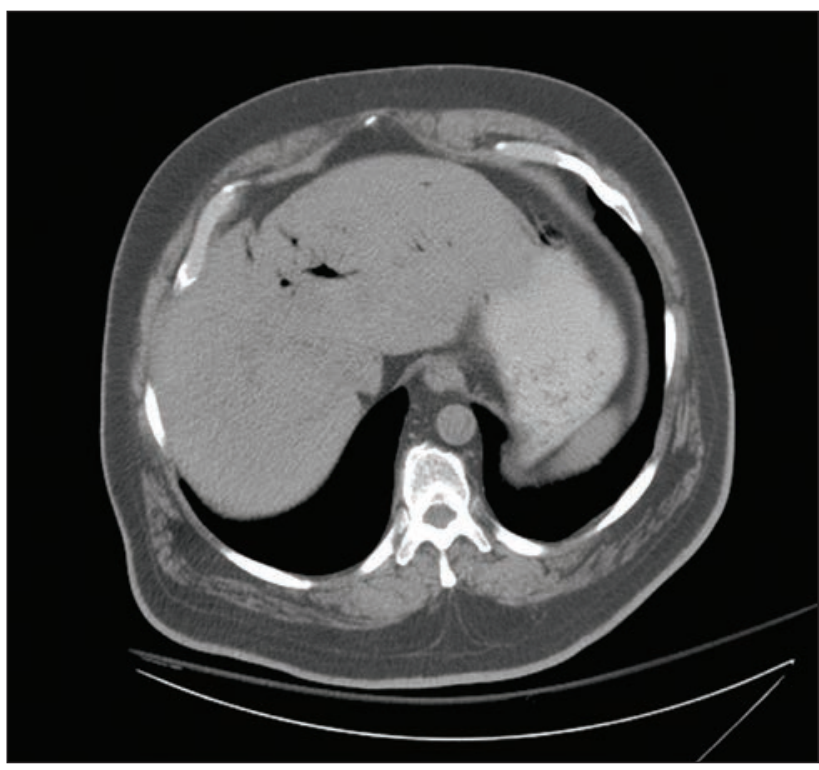

Figure 1. CT scan of the abdomen demonstrating air in the portal venous system as well as in the gastric wall. myocardial infarctions and stent placements), and peptic ulcer disease presented to Thomas Jefferson University Hospital with two weeks of loose stools and two days of nausea, vomiting and diffuse abdominal pain. In the emergency room, the patient had a temperature of $96.8^{\circ} \mathrm{F}$, heart rate of 99 beats per minute, blood pressure of $95 / 58 \mathrm{mmHg}$, respiratory rate of 22 breaths per minute, and oxygen saturation of $96 \%$ on $3 \mathrm{~L}$ of oxygen. His cardiac and respiratory exams were unremarkable. His abdominal exam was notable for hypoactive bowel sounds and pain with deep palpation. He was tympanic to percussion. He did not have peritoneal signs. Labs were significant for a white blood cell count of $11.3 \mathrm{~B} / \mathrm{L}$ (normal range $=4-11 \mathrm{~B} / \mathrm{L}$ ) and lactate of $1.0 \mathrm{mmol} / \mathrm{L}$ (normal range $=0.5-2.2 \mathrm{mmol} / \mathrm{L}$ ) on presentation, which was repeated 6 hours later and found to have increased to $2.5 \mathrm{mmol} / \mathrm{L}$. He underwent a CT scan of the abdomen which revealed a distended stomach with foci of intramural air, with air extending into the portal venous system (Figure 1). Soon after presentation,

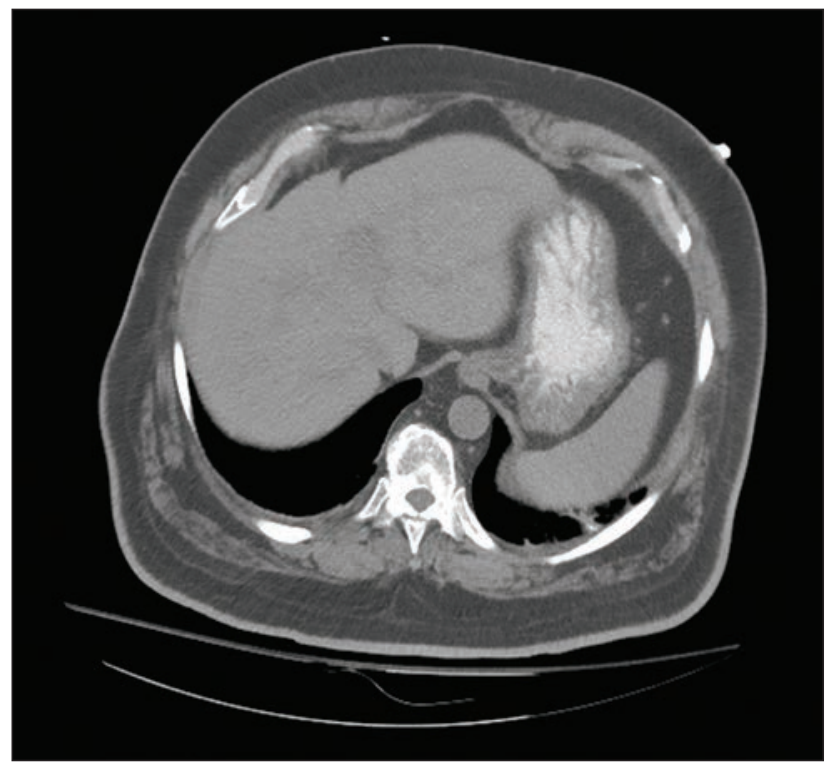

Figure 2. CT scan of the abdomen demonstrating resolution of all air on day 2 of hospitalization 
the patient became hypotensive to a systolic blood pressure of $85 \mathrm{mmHg}$ and was resuscitated with $3 \mathrm{~L}$ normal saline. He was sent to the intensive care unit where he received an additional $2 \mathrm{~L}$ normal saline. His blood pressure responded appropriately. Surgery was consulted regarding the intramural air found on CT scan. However, since the patient did not have an acute abdomen, they deemed that he would not need emergent surgery. It was also decided that an esophagogastroduodenoscopy (EGD) would be too high risk given the increased risk of perforation. He was medically managed with antibiotics and supportive care. He was allowed nothing-by-mouth, pan-cultured, and started on a pantoprazole infusion and broad spectrum antibiotics (vancomycin, aztreonam, and metronidazole.

\section{DIFFERENTIAL DIAGNOSIS}

The significance of radiographic findings showing intramural air within the gastric wall is based entirely on the clinical situation. Intramural air in a patient who recently underwent instrumentation of their gastrointestinal tract significantly differs from intramural air found in an ill, septic patient. The differential for intramural air within the gastric wall includes emphysematous gastritis, as in our case report, versus cystic pneumatosis versus gastric emphysema.

\section{OUTCOME AND FOLLOW UP}

By day 2 of hospitalization, our patient had clinically improved with less abdominal pain and no further vomiting. Blood cultures were negative. A repeat CT scan showed resolution of gas in the stomach wall and portal venous system but showed new wall thickening of the proximal ascending colon (Figure 2). It was thought that the patient had bowel ischemia as a result of the emphysematous gastritis. By day 3 of hospitalization, our patient was restarted on a diet, which he tolerated well. He finished a 10-day course of antibiotics and had complete resolution of all his symptoms. He was discharged home with outpatient gastroenterology follow-up.

\section{DISCUSSION}

Emphysematous gastritis is described as gas in the lining of the stomach wall by gas-producing organisms caused by local spread of the organisms through the mucosa or by hematogenous dissemination from a distant focus. ${ }^{3.5}$ Emphysematous gastritis was first described in 1889 by Frankel who believed it was caused from an infection where a prior insult to the mucosal barrier had occurred, either by corrosives, alcohol abuse, gastric ulcers, abdominal surgery, malignancy or gastroenteritis. ${ }^{4.5}$ Gas within the Gl tract occurs most often in the descending and sigmoid colons, while the stomach is the least common hollow organ for intramural air to occur due to the acidic environment.

Common organisms involved in emphysematous gastritis include Enterobacter species, Pseudomonas aeruginosa, Candida albicans, Staphylococcus aureus, Streptococci, and Escherichia coli. Patients usually present with gastrointestinal signs and symptoms with abdominal pain, distension, bloating, diarrhea, nausea and vomiting. 2.3.6 Diagnosis is usually based on the clinical history, a physical exam suggestive of an acute abdomen and imaging showing gas in the gastric wall and portal venous system, with CT scan being the most sensitive imaging modality. ${ }^{3}$ Characteristic findings include cystic pockets or streaks of air within the gastric wall with thickened mucosal folds, pneumoperitoneum, portal venous gas and occasionally pneumatosis intestinalis. 2,6

Unlike gastric emphysema, which is defined as gas in the stomach lining with no associated infection and has an excellent prognosis with medical management, emphysematous gastritis is rare and has a high mortality rate. Surgery should be avoided during the acute phase in the absence of bowel perforation due to the friability of the mucosa. It is important to treat patients based on their clinical condition and to initiate antibiotics early as mortality rates are as high as $75 \% .{ }^{1}$ Thus, early recognition of the illness, application of broad-spectrum antibiotics and meticulous supportive care are regarded as key therapeutic measures? 


\section{KEY POINTS}

Emphysematous gastritis is described as gas in the lining of the stomach wall caused by gas-producing organisms. It can be caused by an infection where a prior insult to the mucosal barrier has occurred, either by corrosives, alcohol abuse, gastric ulcers, abdominal surgery, malignancy or gastroenteritis. Diagnosis is usually made based on the clinical picture, a physical exam suggestive of an acute abdomen, and imaging showing gas in the gastric wall and portal venous system

\section{REFERENCES}

1. Paul M, John S, Menon M et al. Successful medical management of emphysematous gastritis with concomitant portal venous air: a case report. J of Medical Case Reports, 2010, 4: 140-143.

2. Hadas-Halpren I, Hiller N, Guberman D. Emphysematous gastritis secondary to ingestion of large amounts of Coca Cola. Am J Gastroenterol 1993; 88: 127-9.

3. Tsuan-Hao Loi, Ju-Yaw See, Ravishankar K Diddapur, John R Issac Emphysematous gastritis: a case report and a review of literature. Ann Academy of Medicine 2007; 36: 72-3

4. Van Mook WN, van der Geest S, Goessens ML, Schoon EJ, Ramsay G. Gas within the wall of the stomach due to emphysematous gastritis: case report and review. Eur J Gastroenterol Hepatol 2002; 14: 1155-60

5. Moosvi AR, Saravolatz LD, Wong DH, Simms SM. Emphysematous gastritis: case report and review. Rev Infect Dis 1990; 12: 848-55.

6. Chun-Ta Huang, Wei-Yu Liao. Emphysematous gastritis: A deadly infectious disease. Scandinavian Journal of Infectious Diseases 2009; 41: 317-319

7. Jung JH, Choi HJ, Yoo J, Kang SJ, Lee KY. Emphysematous gastritis associated with invasive gastric mucormycosis: a case report. J Korean Med Sci 2007; 22: 923-7.
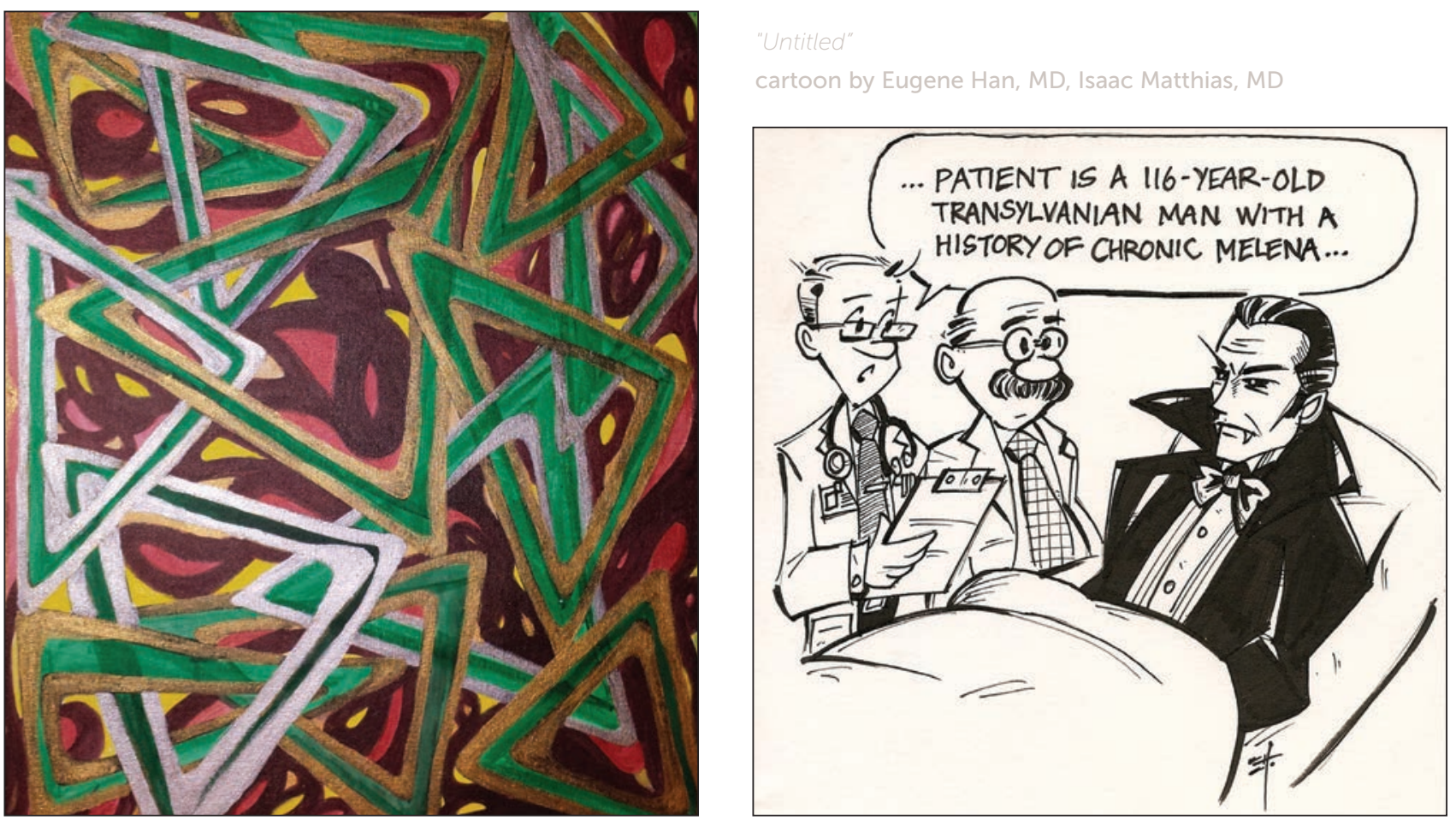